\title{
The Compressional Behavior of Glass-fiber Sheets and the Forming Pressure of FRP
}

\author{
By Kiyohisa Fujino*, Tsuneo Hirai** and Shigehisa Otsuki***, Members, TMSJ \\ * Faculty of Engineering, Kyoto University, Kyoto \\ ** Faculty of Engineering, Osaka City University, Osaka \\ *** Industrial Research Institute, Osaka Prefecture
}

\begin{abstract}
Purpose :

To increase the glass fiber content of FRP which influeces its mechanical character, the glass fiber must be compressed. An investigation has been made into the compressional behavior of a glass fiber pile and its bearing on the relation between the thickness of FRP and the forming pressure.

Results :

Compressional experiments on plain, satin and non-woven glass fibers have shown that a glass fiber pile is compressed by two stages. In the first stage, except under very low pressure, the thickness of the pile diminishes according to the formula $\log t_{0}=-m_{1} \log p+b_{1}$. In the second stage, the thickness of the pile decreases according to the formula $\log t_{0}=-m_{2} \log p+b_{2}$.

Here $m, b$ take different values depending on the state of the cloth and the presence or absence of uncured resin. The experiments have shown also that the relation between the thickness of the lamination and the forming pressure is $p=(n \theta / t)^{1 / m}$. The experiments have also led to the formula $\beta=\beta_{1} p^{m}$ as the relation between the glass fiber content and the forming pressure.
\end{abstract}

\section{Introduction}

To laminate glass fiber-reinforced plastics to a desired thickness by the bag method or by the matched-die method. We have to know how much forming pressure is needed. Otherwise we cannot design the forming press.

A lamination of any desired glass content is obtainable by the matched die method by controlling the number of glass sheets, provided a clearance is given, and the forming pressure is induced on the surface of the die according to number of glass sheets. In the case of the bag method, the glass content of a lamination is fixed mainly by the forming pressure, and the thickness of the lamination is given by the number of sheets.

Therefore, by clarifying the relation among pressure, thickness and glass content, we can build the basis for the forming of FRP and, moreover, know beforehand the strength of FRP while we are doing laminating.

We will investigate the compressional behavior of glass fiber sheets, and go on to study the relation among the forming pressure, the thickness and glass content of a lamination.

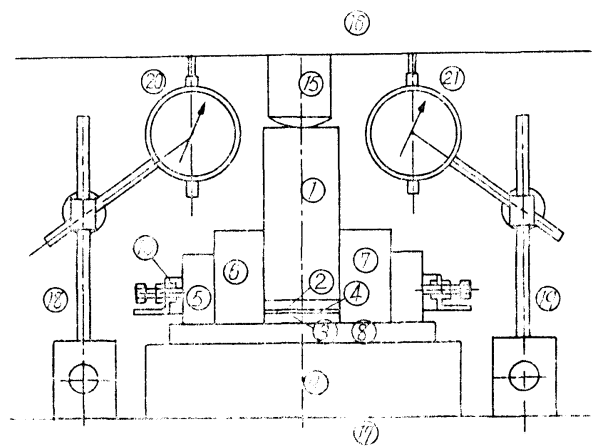

Fig. 1 Experimental instrument to compress glass fiber pile

\section{Compressional Behavior of Glass Fiber Sheets}

(1) Instrument: The experiment was made with the instrument shown in Fig. 1 and which was attached to a $10 \mathrm{~T}$ Amsler oil-type universal testing machine. (4) is the glass fiber sheets placed between 
polished plates (2)(3). They were compressed by (9)(8) 3 and (15)(1)(2) while the testing machine (16)(17) worked. (5) is a case. (6)(7) are guides. (2021) are dial gauges of a $1 / 100 \mathrm{~mm}$ scale, placed $100 \mathrm{~mm}$ from the center. (10) is apparatus to adjust the relative position of (15) and (1)-(8).

(2) Specimens: Three types of glass fiber sheets were used: non-alkalie glass fiber sheet satin (A), alkalie glass fiber sheets, plain woven (B), and non-alkalie glass mats (C). Each sample was cut in size $70 \mathrm{~mm} \times 60 \mathrm{~mm}$, and 30 cut pieces made into one specimen.

(3) Experiment: The relative position of (15) and (1) - (8) was so adjusted that the specimens (4) were compressed uniformly. Then a desired pressure was given them by the testing machine. The reduction in height corresponding to pressure began to be read on the dial gauges when (ib) contacted (1). We read the indication after compressing the specimens for 3 minutes, because the height of the specimen remained unchanged even after 2 minutes.

(4) Results of experiment: Fig. 2 shows the results of the experiment on specimen $A$. The abscissa was given by the common logarithm of the compression pressure on the sheet $\log _{10} p$. The ordinate was given by the common logarithm of the thickness of one sheet $\log _{10} t_{0}$.

The figure shows that the results of the measurement are plotted along two straight lines having different inclinations, and that these relations hold good for specimens $B$ and $C$ also.

The relations are expressible by this formula:

$$
\begin{aligned}
& \log t_{0}=-m_{1} \log p+b_{1} \\
& \log t_{0}=-m_{2} \log p+b_{2}
\end{aligned}
$$

(1) is the formula for the first stage, (2) for the second stage. The constants in these formulas

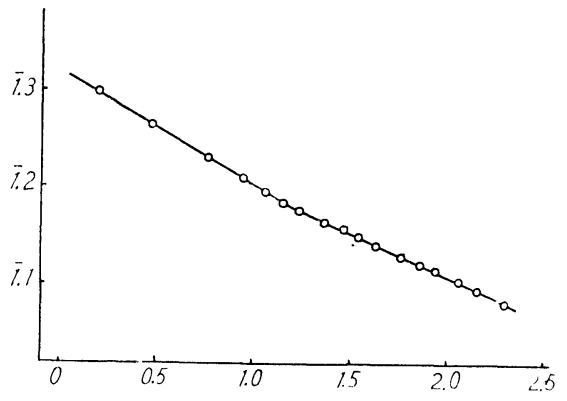

Fig. 2 Relation between thickness of dry glass cloth of cloth $A$ and compression pressure

ord. thickness of glass cloth $\mathrm{mm}$ (log.)

abs. pressing pressure $\mathrm{kg} / \mathrm{cm}^{2}$ (log.)

and the calculated pressure at the intersecting point are given in Table 1 , as an example. $D, W, N, R$, respectively, mean dried, wetted and natural glass fiber sheets used without and with resin.

(5) Discussion: To investigate the behavior of glass fiber sheets during the process of compression, plain woven glass fiber sheets were set with polyester resin in a state of compression and photographed with a magnifying lens. Figs. 3 and 4 show the so-called first stage and Figs. 5 and 6 the second stage.

It was observed from the photographs that, in the first stage, the cross sections of the fiber bundles were deformed, in that they decreased in height and spread sideways, because a glass fiber itself is rigid and its elastic modulus is large.

Therefore, with the decrease in the height of a bundle, the points of contact between pieces of glass cloth increase. So does the total spring constant, i.e., elastic deformation resistance.

\begin{tabular}{|c|c|c|c|c|c|c|c|}
\hline & & Before & point & After & point & & cross point \\
\hline Sat & & $\begin{array}{c}\text { Coefficient } \\
m_{1}\end{array}$ & $\begin{array}{c}\text { Constant } \\
b_{1}\end{array}$ & $\begin{array}{c}\text { Coefficient } \\
m_{2}\end{array}$ & $\begin{array}{l}\text { Constant } \\
b_{2}\end{array}$ & $\log _{11} P_{k}$ & $\begin{array}{l}\text { Compression press. } \\
\qquad p_{k} \mathrm{~kg} / \mathrm{cm}^{2}\end{array}$ \\
\hline Cloth $A$ & $D A$ & 0.1165 & -0.6785 & 0.0897 & -0.7101 & 1.22 & 16.5 \\
\hline & $W A$ & 0.1190 & -0.6800 & 0.0974 & -0.7054 & 1.18 & 15.1 \\
\hline & $R A$ & 0.0967 & -0.7373 & 0.0743 & -0.7586 & 0.96 & 9.1 \\
\hline & $D B$ & 0.1257 & -0.6704 & 0.1119 & -0.6777 & 0.53 & 3.4 \\
\hline Cloth $B$ & $W B$ & 0.1264 & -0.6791 & 0.1138 & -0.6835 & 0.35 & 2.2 \\
\hline & $R B$ & 0.1281 & -0.6968 & 0.0997 & -0.7151 & 0.64 & 4.4 \\
\hline Cloth $C$ & $N C$ & 0.1405 & -0.2954 & 0.1029 & -0.3358 & 1.07 & 11.7 \\
\hline & $R C$ & 0.0933 & -0.5535 & 0.0859 & -0.5584 & 0.88 & 7.6 \\
\hline
\end{tabular}

Table 1 Examples of Experimental Results 


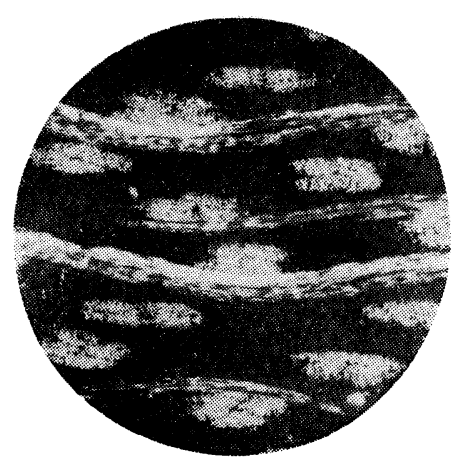

Fig. 3

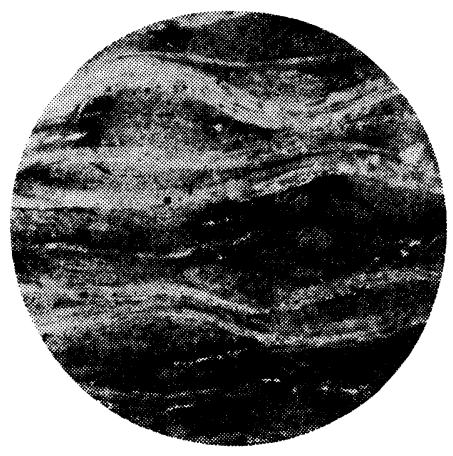

Fig. 5

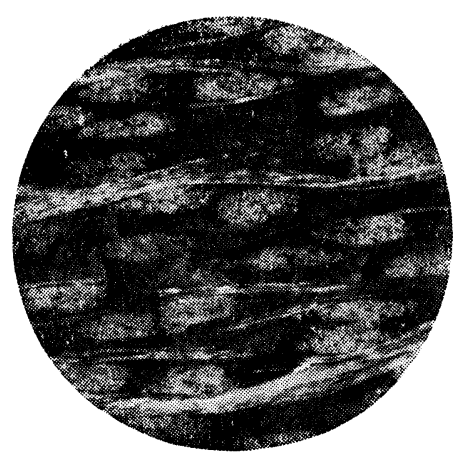

Fig. 4

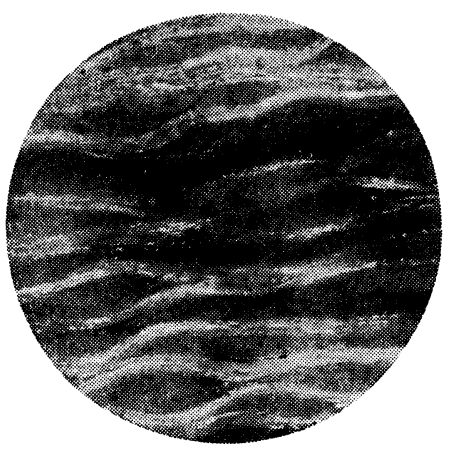

Fig. 6

Fig. 3 $\mathbf{6}$ Magnified photographs of compressed glass cloth pile

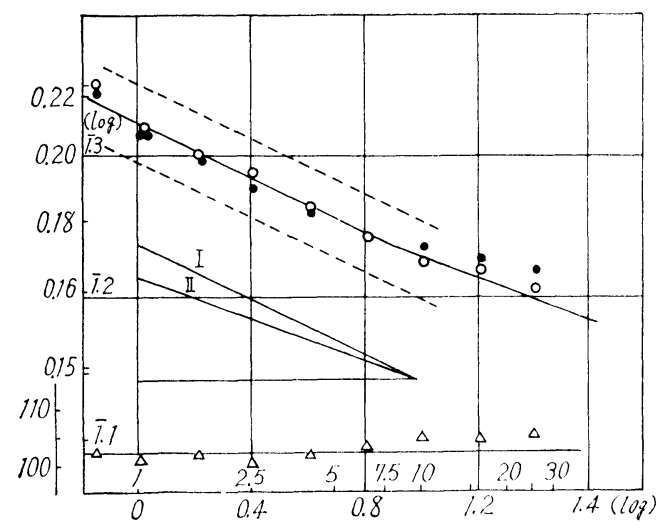

Fig. 7 Relation between forming pressure and thickness of FRP using cloth $A$

ord. thickness of FRP per sheet of glass cloth $\mathrm{mm}$ weight of a sheet of laminated glass cloth $(\mathrm{g})$

abs. forming pressure $\mathrm{kg} / \mathrm{cm}^{3}$

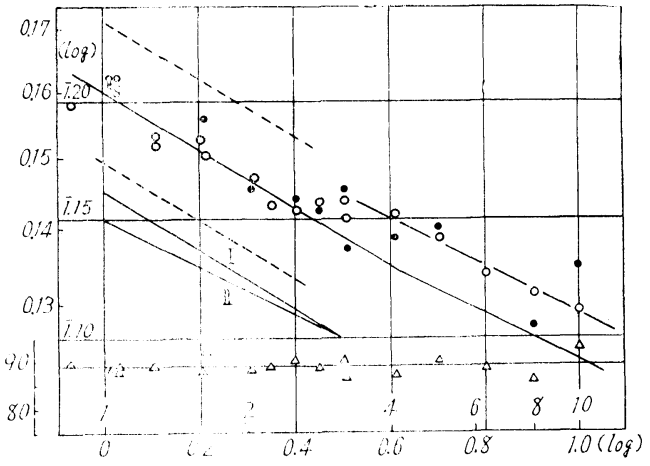

Fig. 8 Relation of forming pressure and thickness of FRP using cloth $B$

ord. thickness of FRP per sheet of glass cloth $\mathrm{mm}$ weight of a sheet of laminated glass cloth $(\mathrm{g})$

abs. forming pressure $\mathrm{kg} / \mathrm{cm}$ : 
The restraining force, which is another factor in resistance to deformation, is induced by friction between filaments. It is assumed, therefore, that the restraining force increases approximately in proportion to the pressing pressure $(p)$; and that deformation resistance in the glass fiber layers is affected mutually by these things, as the formula shows :

$$
\begin{gathered}
K=-\frac{\Delta p}{\Delta t}=k_{1} \frac{p}{t} \\
\log t=\frac{1}{k_{1}} \log p+c_{1}
\end{gathered}
$$

If height $t$ is replaced with $t_{0}$ which means the thickness of a glass fiber sheet, and if the coefficient is also changed, then formula (4) will be the same as formula (1).

From the magnified photographs we can understand that the criss-crossing of fiber bundles, contact between one bundle with another and twistimposed restraint made it difficult for the cross section of a fiber bundle to be deformed at the end of the first stage, consequently the regular wave of the fiber bundles was disturbed and the bundles shifted either to the open space near the crossing point, or the open space created by lack of the fiber bundle.

Accordingly, although the proportional coefficient differs somewhat from that in the first stage, the deformation resistance induced by an elastic or frictional factor is proportional to the compressed pressure and is in inverse proportion to the thickness of the layers, to wit:

$$
K=k_{2} \frac{p}{t}
$$

Therefore, formula (2), like formula (4), is given for the second stage.

\section{Forming Pressure of $F R P$}

The foregoing has explained that the thickness of glass fiber sheets of the same kind is diminished by compression, the formula of diminition being $\log t_{0}=-\mathrm{m} \log p+b$. The layers of compressed glass fiber sheets, however, differ in thickness according to the kind and number of the sheets.

What follows is an investigation of whether the formula holds good as to the forming of FRP and of the relation between forming pressure and the glass fiber content which influences the strength of FRP.

\section{(1) Metod of experiment}

Polyester mixed with a catalyzer and a promoter was poured into a mold coated with separating agent.

As air bubbles were removed, glass fiber sheets were dipped, one by one, in the mixture. Then they were subjected to pressure-reducing in a sealed vessel to remove bubbles.

The samples were compressed by an Amsler universal testing machine with uniform pressure and were cured by electric heating plates and with ultrared lamps.

After curing and cooling, the thickness of the formed samples was measured with a micrometer. The specimens were made of three kinds of glass fiber sheets, $A, B$ and $C$, and polyester Epolac $G$.

$A$ is a non-alkalie glass fiber satin woven cloth surface-treated with volan. $B$ is an alkalie glass fiber plain woven cloth treated with silan. $C$ is a non-alkalie glass fiber mat treated with volan. They were cut in size $140 \mathrm{~mm} \times 170 \mathrm{~mm}$. We used 40 sheets of $A, 50$ of $B$ and 25 of $C$, so that the finished thickness was about $7 \mathrm{~mm}$.

The glass fiber sheets used were weighed or their thickness was measured for the calibration of data.

\section{(2) Result}

On the assumption that the formula just described was tenable, the measured values were plotted in the following figures which had log-log coordinate. The ordinate is for the mean thickness of the FRP lamination per sheet of glass cloth. The abscissa is for the forming pressure.

\section{(i) Results on $A$}

Fig. 7 gives the results on cloth $A$. The thickness of the formed specimen was corrected according to the weight of the glass cloth used. The black marks show the non-corrected values. The white marks show the corrected values. The triangular marks show the weight of the laminated glass cloth, the value of which was used for correction. The straight line means the average of the weights.

The inclination I, II shows the coefficient $-m_{1}$, $-m_{2}$ obtained by the above-mentioned pressing experiment on polyester-soaked cloth $A$. The full lines which cut through the dots have the same inclination as $-m_{1},-m_{2}$. The broken line show $\log (t / n)=-m_{1} \log p+b \pm b_{c}$, where added constant $\pm b_{e}$ is the value obtained by multiplying the coefficient of variant, which is obtained for the weight 
of $A$ sample, by constant $b_{1}$. The value of the slightly bent point shown in the figure is the same value as is obtained by the pressing experiment on polyester-soaked cloth $A$.

The foregoing shows that the relation between the thickness of FRP, reinforced with material $A$, and the forming pressure is not different from the $p-t$ relation if polyester-soaked cloth $A$ is compressed. Note, however, that the thickness of FRP has to be corrected according to the unevenness of the glass cloth. Remember also that, under a forming pressure higher than that on the slightly bent point, the thickness of FRP deviates from the line obtained by the compressing experiment.

(ii) Results on cloth $B$

Fig. 8 shows the experimental results on FRP made of cloth $B$ and polyester. Here again, because it was not possible to avoid that error in the laminated sample which is caused by the unevenness of the reinforcing material, the measured amount was corrected as in the case of (i).

The explanations about the various marks in Fig. 7 apply here also.

Note in connection with Fig. 8, however, that the corrected experimental values in the second stage are plotted beside the chain line having an inclination of $-m_{2}$. Indeed, they changed their course and headed for this line at the end of the first stage.

The reason for this fact is presumably this: Polyester resin which enters the glass cloth is squeezed out by pressing force, setting takes while the adhesive force between cloths diminishes and the lamination is expanded by the elasticity of the glass fiber when the pressure is removed.

Such a phenomenon, it seems, is likely to occur in any reinforcing material, and when it does occur, the measured values tend to deviate upward from the line of the second stage. Therefore, the $p-t$ relation should be applied in the first stage where the adhesive force of resin is good. Of course, the lamination is useless if adhesion is poor. In that case the formula for the second stage is not needed.

Fig. 9 shows the results on FRP reinforced with cloth $C$. We notice here, too, that the relation between the thickness of FRP and the forming pressure is the same as in the two cases cited earlier.

(3) Formulas concerning the factors in the forming of FRP

So long as a lamination is used for practical purposes, the formula on the relation between the thickness and forming pressure of FRP is:

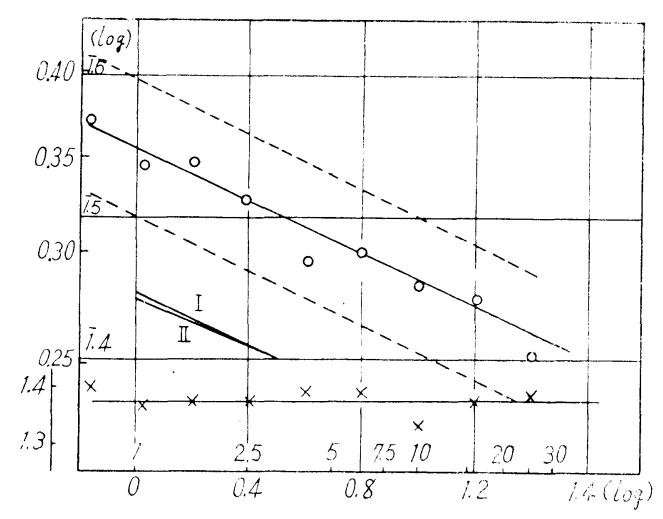

Fig. 9 Relation of forming pressure and thickness of FRP using cloth $C$

ord. thickness of FRP per sheet of glass cloth $\mathrm{mm}$ thickness of a sheet of laminated mat $\mathrm{mm}$

abs. forming pressure $\mathrm{kg} / \mathrm{cm}^{2}$

$$
\log (t / n)=-m \log p+b
$$

which is rewritten thus:

$$
t p^{m} / n=\log ^{-1} \quad b \equiv \theta
$$

so that, when $p=1 \mathrm{~kg} / \mathrm{cm}^{2}$ and $t=t_{1}$, then :

$$
\theta=t_{1} / n
$$

Accordingly, $\theta$ means the thickness of a resinsoaked glass fiber sheet under $1 \mathrm{~kg} / \mathrm{cm}^{2}$. If we take $t_{a}$ as the thickness of a resin-soaked glass fiber sheet under any pressure $p_{a}$, then the coefficient $m$ is given as $m=\left(\log t_{1}-\log t_{a}\right) / \log p_{a}$

This coefficient $m$ should be determined experimentally for each reinforcing material, because it differs according to the construction and the condition of the surface of the glass fiber sheet and the nature of the resin used. Thus, where $m$ and $\theta$ of a reinforcing material is known, the pressure needed for forming is given by following formula which is formula (7) rewritten:

$$
p=\left(\frac{n \theta}{t}\right)^{\stackrel{1}{m}}
$$

However, the number of the glass fiber sheets for lamination should be calculated by this formula[1]:

$$
n / t=\beta \rho_{g} / w
$$

where $\beta$ is the glass fiber content, in volume, of FRP. $\rho_{g}$ is the specific gravity of the glass fiber and $w$ is the weight of a glass fiber sheet per unit area.

The mechanical characteristics of FRP are affected by the content of the material which reinforces the glass fiber and the relation between the 
content of the glass fiber and the forming pressure is obtainable from formulas (10) and (11), thus:

$$
p=\left(\theta \beta \rho_{g} / w\right)^{1 / m}
$$

$\theta, m, w, \rho_{g}$ being values peculiar to the reinforcing material, the forming pressure should be the function of the glass fiber content, in volume, of lamination. Therefore, if $\beta_{1}$ is the glass fiber content in volume at $1 \mathrm{~kg} / \mathrm{cm}^{2}$, the coefficient of $\beta^{1 / m}$ is $1 /\left(\beta_{1}\right)^{1 / m}$ and the forming pressure of FRP are decided by the glass fiber content and the kinds of the reinforcing material and the resin.

\section{Conclusions}

Compressional experiments have made it clear that piles of three kinds of glass fiber sheets all diminish in thickness according to the formula $\log t_{0}=-m_{1} \log p+b_{1}$ in the first stage, and according to $\log t_{0}=-m_{2} \log p-b_{2}$ in the second stage. If the results of the experiment on the resin-soaked reinforcing material are used for $m_{1}$ and $b_{1}$, then the thickness of FRP also follows these formulas.
However, FRP gets poor with the squeezing out of resin from the glass fiber in the compressional process in the second stage and, in this condition, is not suitable for practical use. The formula on the forming pressure and the thickness of FRP, then, is obtainable by developing the formula for the first stage into $p=(n \theta / t)^{1 / n}$.

The relation between the thickness of $F R P$ and the number of glass fiber sheets for lamination is given by the formula $n=t \beta \rho_{g} / w$.

To make a stronger FRP, the forming pressure must be increased to obtain a higher glass fiber content.

(1) Number of sheets from the reinforce, (2) forming pressure, (3) required thickness, (4) required grass fiber content of FRP, and also (5) the capacity of forming press are obtainable from the above formulas.

\section{Literature}

[1] S. Otsuki; Reports of Ind. Res. Inst. Osaka Pref., 19, 9 (1958) 